\title{
Prävention zwischen sinnvoller Steuerung und Bevormundung
}

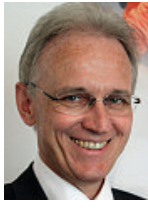

Hans-Ulrich Bigler

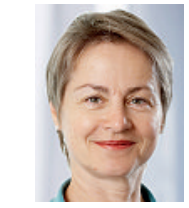

Christine Romann

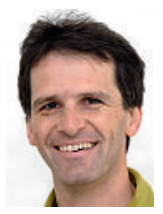

Roy Salveter
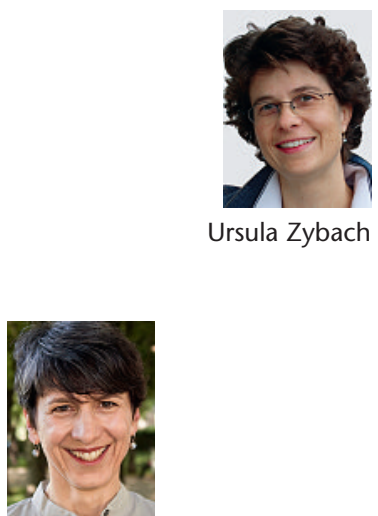

Anna Sax
«Vorbeugen ist besser als heilen», sagt der Volksmund. Wer würde dem nicht zustimmen? Wenn es aber darum geht, wo Gesundheitsförderung und Prävention ansetzen und wer dafür zuständig sein soll, scheiden sich die Geister, wie die Diskussionen um das Präventionsgesetz zeigen. Während von den Befürwortern der Vorlage die Notwendigkeit und der Nutzen einer national koordinierten Strategie mit klaren Gesundheitszielen betont werden, befürchten die Gegner eine Bevormundung der mündigen Bürger durch den Staat und setzen weitgehend auf Eigenverantwortung.

\section{Diskutieren Sie mit}

Braucht es im Präventionsbereich tatsächlich neue Steuerungs- und Koordinationsinstrumente, wie sie im Entwurf des Präventionsgesetzes vorgesehen sind? Erhöht eine national koordinierte Prävention und

\section{Die Podiumsgäste}

Unter der Leitung von Anna Sax, lic. oec. publ., MHA, Redaktorin der Schweizerischen Ärztezeitung und selbständige Gesundheitsökonomin, diskutieren:

- Hans-Ulrich Bigler, Direktor Schweizerischer Gewerbeverband

- Dr. med. Christine Romann, Mitglied des Zentralvorstands der FMH, Verantwortliche
Gesundheitsförderung die gesamtwirtschaftliche Produktivität, oder verursacht sie durch Aufblähung der Administration lediglich zusätzliche Kosten, wie Wirtschaftskreise befürchten? In welchen Bereichen ist eine behördlich gesteuerte Gesundheitsförderung und Prävention sinnvoll, wann beschneidet sie die Freiheitsrechte des Individuums in unzulässiger Weise?

$\mathrm{Zu}$ diesem Themenkreis diskutiert am nächsten Podiumsanlass der Schweizerischen Ärztezeitung eine profilierte Vertreterin der Ärzteschaft mit Fachleuten aus Wissenschaft, Wirtschaft und Verwaltung. Der Einbezug des Publikums in die Diskussion ist zentraler Bestandteil des Konzepts dieser Veranstaltungen, mit denen ein interessanter und konstruktiver Beitrag zur Debatte aktueller Fragen des Schweizer Gesundheitswesens geleistet werden soll.
Die Veranstaltung wird in Zusammenarbeit mit der Ärztegesellschaft des Kantons Bern organisiert.

Die Durchführung des Anlasses wird möglich dank grosszügiger Unterstützung durch Interpharma, den
Ressort Gesundheitsförderung und Prävention

- Dr. phil. nat. Roy Salveter, Bundesamt für Gesundheit, Co-Leiter Abteilung Nationale Präventionsprogramme

- Ursula Zybach, dipl. Lm.-Ing. ETH, Präsidentin Public Health Schweiz, Mitglied der Geschäftsleitung der Krebsliga Schweiz
Verband der forschenden pharmazeutischen Industrie. Die Verantwortung für Konzept und Inhalt des Podiums liegt bei der Schweizerischen Ärztezeitung.

\section{Eintritt frei - Anmeldung erforderlich}

Die öffentliche Podiumsdiskussion mit anschliessendem Apéro findet statt am Mittwoch, 6. Juli 2011, 19-21 Uhr im Empire-Saal des Restaurants Zum Äusseren Stand, Zeughausgasse 17, Bern. Der Eintritt ist frei, eine Anmeldung ist jedoch erforderlich. Diese kann bis Freitag, 1. Juli, via E-Mail an redaktion.saez@emh.ch oder via Fax an 0614678556 erfolgen. Bitte Ihren Namen und die Namen allfälliger Begleitpersonen sowie das Stichwort «Anmeldung zum SÄZ-Podium vom 6. Juli» angeben. Auch telefonische Anmeldungen sind vormittags unter 0614678572 möglich. 